T. Matsuzawa

Nagoya Math. J.

Vol. 51 (1973), 57-77

\title{
ON SOME DEGENERATE PARABOLIC EQUATIONS I
}

\author{
TADATO MATSUZAWA
}

\section{§1. Introduction}

Let $\Omega, I$ be open intervals in $R_{x}=(-\infty<x<\infty), R_{t}=(-\infty<t$ $<\infty)$ respectively. For a function $a(x, t) \in C^{\infty}(\Omega \times I)$, consider the partial differential operator

$$
L=\frac{\partial}{\partial t}+a(x, t) \frac{\partial}{\partial x} .
$$

A sufficient condition of Nirenberg and Treves (cf. [7]) for the operator (1.1) to be hypoelliptic ${ }^{1)}$ in $\Omega \times I$ is expressed as follows:

(1.2) for all $x \in \Omega$, the function $t \rightarrow \operatorname{Im} a(x, t)$ has only zeros of even order less than or equal to $2 \ell$ in the interval $I$. ( $\ell$ interger, $\geqq 0$ ).

This is necessary and sufficient condition when $a(x, t)$ is analytic in $\Omega \times I$.

Motivated by this fact, we shall consider the hypoellipticity of a degenerated parabolic operator defined in $\Omega \times I$ :

$$
P=\frac{\partial}{\partial t}-a(x, t) \frac{\partial^{2}}{\partial x^{2}}+b(x, t) \frac{\partial}{\partial x}+c(x, t),
$$

where $a(x, t), b(x, t), c(x, t) \in C^{\infty}(\Omega \times I)$ and satisfy the following conditions.

$$
\operatorname{Re} a(x, t) \geqq 0 \quad \text { in } \Omega \times I,
$$

(1.5) for all $x \in \Omega$, the function $t \rightarrow \operatorname{Re} a(x, t)$ has only zeros of even order less than or equal to $2 \ell$ in the interval $I$,

$$
|\operatorname{Im} a(x, t)| \leqq C \operatorname{Re} a(x, t) \quad \text { in } \Omega \times I,
$$

Received December 11, 1972.

1) The operator $P$ is said to be hypoelliptic, if any distribution $u$ is infinitely differentiable in every open set where $P u$ is infintely differentiable. 


$$
\begin{gathered}
\left|\operatorname{Im} a_{x}(x, t)\right| \leqq C[\operatorname{Re} a(x, t)]^{1 / 2} \quad \text { in } \Omega \times I, \\
|b(x, t)| \leqq C[\operatorname{Re} a(x, t)]^{1 / 2} \quad \text { in } \Omega \times I,
\end{gathered}
$$

where $C$ denotes a positive constant.

Our aim is to construct the parametrices of the operator $P$. To do so, we shall mainly rely upon the procedure of [4], [5] and the theory of pseudo-differential operators developped in [1], [2]. Main result is the following.

THEOREM 1.1. Suppose the operator (1.3): $P$ satisfies the conditions (1.4) $\sim$ (1.8) then the operator $P$ is hypoelliptic in $\Omega \times I$.

EXAMPLE. The operator

$$
\frac{\partial}{\partial t}-\left(t^{2 \ell}+x^{2 m}\right) \frac{\partial^{2}}{\partial x^{2}}+i\left(t^{\ell}+x^{m}\right) \frac{\partial}{\partial x}+1, \ell, m \text { integers }>0,
$$

satisfies the conditions $(1.4) \sim(1.8)$ in a neighborhood of the origin.

At the end of $\S 6$, we shall give the other examples of the operators for which our method can be applied.

\section{§ 2. Preliminary lemmas}

We prepare the useful lemmas derived from the recent results of Nirenberg and Treves.

Lemma 2.1. (cf. [7], Lemma 3.1 and [6], part I). Under the conditions (1.4) and (1.5), for any compact set $K \subset \Omega \times I$ there exists a constant $C>0$ such that

$$
\left|\operatorname{Re} a_{x}(x, t)\right| \leqq C(\operatorname{Re} a(x, t))^{1 / 2} \quad(x, t) \in K .
$$

Lemma 2.2. (cf. [7], Lemma $C .1, \ldots$ ) Under the conditions (1.4) and (1.5), for any compact set $K \subset \Omega \times I$ there exists a constant $\delta>0$ such that

$$
\delta\left(t-t^{\prime}\right)^{2 \ell+1} \leqq \operatorname{Re} \int_{t^{\prime}}^{t} a(x, \tau) d \tau,
$$

where $(x, t),\left(x, t^{\prime}\right) \in K$ and $t^{\prime} \leqq t$.

\section{§3. Formal construction of the parametrices}

Our aim is to construct approximate solutions (parametrices) of the equation 


$$
P_{x, t} E\left(x, y, t, t^{\prime}\right)=\delta\left(x-y, t-t^{\prime}\right) .
$$

For $v\left(y, t^{\prime}\right) \in C_{0}^{\infty}\left(R_{y, t^{\prime}}^{2}\right)$ we set

$$
\hat{v}\left(\xi, t^{\prime}\right)=(2 \pi)^{-1 / 2} \int e^{-i y \xi} v\left(y, t^{\prime}\right) d y, \quad \xi \in R_{\xi} .
$$

Assume a parametrix of $P$ can be written in the following form

$$
[\mathscr{K} v](x, t)=\int_{T_{1}}^{t}\left(\int_{-\infty}^{\infty} e^{i x \xi} K\left(x, \xi ; t, t^{\prime}\right) \hat{v}\left(\xi, t^{\prime}\right) d \xi\right) d t^{\prime}
$$

for $v\left(y, t^{\prime}\right) \in C_{0}^{\infty}(\Omega \times I)$ and $(x, t) \in \Omega \times I$. Hereafter we put $I=\left(T_{1}<\right.$ $t<T_{2}$ ). We have, by a formal computation,

$$
\begin{aligned}
P[\mathscr{K} v](x, t)= & \int_{T_{1}}^{t} \int_{-\infty}^{\infty} e^{i x \xi}\left[\frac{\partial}{\partial t}-a(x, t)\left(\frac{\partial}{\partial x}+i \xi\right)^{2}\right. \\
& \left.+b(x, t)\left(\frac{\partial}{\partial x}+i \xi\right)+c(x, t)\right] K\left(x, \xi ; t, t^{\prime}\right) \hat{v}\left(\xi, t^{\prime}\right) d \xi d t^{\prime} \\
& +\left.\int_{-\infty}^{\infty} e^{i x \xi} K\left(x, \xi ; t, t^{\prime}\right) \hat{v}\left(\xi, t^{\prime}\right) d \xi\right|_{t^{\prime}=t}
\end{aligned}
$$

from where we arrive at the following Cauchy problem:

$$
\begin{gathered}
{\left[\frac{\partial}{\partial t}-a(x, t)\left(\frac{\partial}{\partial x}+i \xi\right)^{2}+b(x, t)\left(\frac{\partial}{\partial x}+i \xi\right)+c(x, t)\right]} \\
\cdot K\left(x, \xi ; t, t^{\prime}\right)=0 \quad \text { in } \Omega \times R_{\xi} \times \Delta, \\
\Delta \equiv\left\{\left(t, t^{\prime}\right) ; T_{1}<t^{\prime}<t<T_{2}\right\} \\
\left.K\left(x, \xi ; t, t^{\prime}\right)\right|_{t=t^{\prime}}=1 \\
K\left(x, \xi ; t, t^{\prime}\right) \mid=0 \quad \text { if } t^{\prime}>t .
\end{gathered}
$$

As a first approximation of the solution of this problem we take $K_{0}(x, \xi$; $\left.t, t^{\prime}\right)$ as follows:

$$
\begin{gathered}
L_{1} K_{0}=\left[\frac{\partial}{\partial t}+a(x, t) \xi^{2}\right] K_{0}\left(x, \xi ; t, t^{\prime}\right)=0 \quad \text { in } \Omega \times R_{\xi} \times \Delta, \\
\left.K_{0}\left(x, \xi ; t, t^{\prime}\right)\right|_{t=t^{\prime}}=1, \\
K_{0}\left(x, \xi ; t, t^{\prime}\right) \mid=0 \quad \text { if } t<t^{\prime} .
\end{gathered}
$$

The solution $K_{0}$ can be written explicitely: 


$$
K_{0}\left(x, \xi ; t, t^{\prime}\right)=\left\{\begin{array}{c}
\exp \left(-\int_{t^{\prime}}^{t} a(x, \tau) \xi^{2} d \tau\right) \quad \text { in } \Omega \times R_{\xi} \times \bar{\Delta} \\
\bar{\Delta} \equiv\left\{\left(t, t^{\prime}\right) ; T_{1}<t^{\prime} \leqq t<T_{2}\right\} \\
\quad \text { if } t<t^{\prime}
\end{array}\right.
$$

Next we set

$$
\begin{aligned}
L_{2}= & -2 i \xi a(x, t) \frac{\partial}{\partial x}-a(x, t) \frac{\partial^{2}}{\partial x^{2}}+b(x, t) \frac{\partial}{\partial x} \\
& +i \xi b(x, t)+c(x, t) \quad \text { in } \Omega \times R_{\xi} \times I .
\end{aligned}
$$

We define $K_{j}\left(x, \xi ; t, t^{\prime}\right), j=0,1,2, \cdots$, recurcively as the solutions of the following problem:

$$
\begin{gathered}
{\left[\frac{\partial}{\partial t}+a(x, t) \xi^{2}\right] K_{j+1}\left(x, \xi ; t, t^{\prime}\right)=-L_{2} K_{j}\left(x, \xi ; t, t^{\prime}\right)} \\
\text { in } \Omega \times R_{\xi} \times \Delta, \\
\left.K_{j+1}\left(x, \xi ; t, t^{\prime}\right)\right|_{t=t^{\prime}}=0 \\
K_{j+1}\left(x, \xi ; t, t^{\prime}\right)=0 \quad \text { if } t<t^{\prime} .
\end{gathered}
$$

Apparently we have

$$
\begin{aligned}
K_{j+1}\left(x, \xi ; t, t^{\prime}\right)= & -\int_{t^{\prime}}^{t} \exp \left(-\int_{t^{\prime}}^{t} a(x, \tau) \xi^{2} d \tau\right) L_{2} K_{j}\left(x, \xi ; s, t^{\prime}\right) d s \\
= & -\int_{t^{\prime}}^{t} K_{0}(x, \xi ; t, s) L_{2} K_{j}\left(x, \xi ; s, t^{\prime}\right) d s \\
& \quad \text { in } \Omega \times R_{\xi} \times \bar{\Delta} .
\end{aligned}
$$

According to the formula (3.2), we set

$$
\begin{gathered}
{\left[\mathscr{K}_{j} v\right](x, t)=\int_{T_{1}}^{t}\left(\int_{-\infty}^{\infty} e^{i x \xi} K_{j}\left(x, \xi ; t, t^{\prime}\right) \hat{v}\left(\xi, t^{\prime}\right) d \xi\right) d t^{\prime},} \\
j=0,1,2, \cdots,
\end{gathered}
$$

for $v\left(y, t^{\prime}\right) \in C_{0}^{\infty}(\Omega \times I)$. A direct computation shows that

$$
\begin{aligned}
& P\left[\left(\mathscr{K}_{0}+\mathscr{K}_{1}+\cdots+\mathscr{K}_{j}\right) v\right](x, t) \\
& \quad=v(x, t)+\int_{T_{1}}^{t} \int_{-\infty}^{\infty} e^{i x \xi} L_{2} K_{j}\left(x, \xi ; t, t^{\prime}\right) \hat{v}\left(\xi, t^{\prime}\right) d \xi d t^{\prime}
\end{aligned}
$$

Symbolically we have

$$
P\left(\mathscr{K}_{0}+\mathscr{K}_{1}+\cdots+\mathscr{K}_{j}\right)=\delta\left(x-y, t-t^{\prime}\right)+L_{2} \mathscr{K}_{j} \cdot
$$


This formula suggests us the method of construction of the parametrices. We shall study the properties of $K_{j}\left(x, \xi ; t, t^{\prime}\right)$ as a symbol of a pseudodifferential operator with parameter $\left(t, t^{\prime}\right)$.

\section{§4. Principal symbol}

$K_{0}\left(x, \xi ; t, t^{\prime}\right)$.

We recall some notations:

$$
\begin{aligned}
& \Omega \text { an open interval in } R_{x}=(-\infty<x<\infty), \\
& I=\left(T_{1}<t<T_{2}\right), \\
& \Delta=\left\{\left(t, t^{\prime}\right) ; T_{1}<t^{\prime}<t<T_{2}\right\} \\
& \bar{\Delta}=\left\{\left(t, t^{\prime}\right) ; T_{1}<t^{\prime} \leqq t<T_{2}\right\}
\end{aligned}
$$

DEFINITION 4.1. (cf. [2], Definition 1.1.1.) Let $m, \rho, \delta$ be real numbers with $0 \leqq \rho \leqq 1,0 \leqq \delta \leqq 1$. Then we denote by $S_{\rho, \delta}^{m}\left(\Omega \times R_{\xi}\right)$ the set of all $a=a(x, \xi) \in C^{\infty}\left(\Omega \times R_{\xi}\right)$ such that for every compact set $K \subset \Omega$ and all integers $\alpha, \beta(\geqq 0)$ the estimate

$$
\left|D_{x}^{\beta} D_{\xi}^{\alpha} a(x, \xi)\right| \leqq C_{\alpha, \beta, K}(1+|\xi|)^{m-\rho \alpha+\delta \beta}, x \in K, \xi \in R_{\xi}^{1},
$$

is valid for some constant $C_{\alpha, \beta, K}$. The elements of $S_{\rho, \delta}^{m}$ are called symbols of order $m$ and type $\rho, \delta$. We set

$$
S^{-\infty}=S_{\rho, \delta}^{-\infty}=\bigcap_{m} S_{\rho, \delta}^{m} .
$$

Obviously $S_{\rho, \hat{o}}^{m}\left(\Omega \times R_{\xi}\right)$ is a Fréchet space with the topology defined by taking as seminorms the best constants $C_{\alpha, \beta, K}$ in (4.1).

Definition 4.2. Let $\Lambda$ be a subset of $R_{t} \times R_{t^{\prime}}$. We denote by $\mathscr{E}^{0}\left(\Lambda ; S_{\rho, \delta}^{m}\left(\Omega \times R_{\xi}\right)\right)$ the set of all $K\left(x, \xi ; t, t^{\prime}\right)$ such that

$$
K\left(x, \xi ; t, t^{\prime}\right) \in S_{\rho, \delta}^{m}\left(\Omega \times R_{\xi}\right)
$$

for every $\left(t, t^{\prime}\right) \in \Lambda$ and continuous with respect to parameter $\left(t, t^{\prime}\right)$ in 1. For an integer $p \geqq 0$ we say that

$$
K\left(x, \xi ; t, t^{\prime}\right) \in \mathscr{E}^{p}\left(\Lambda ; S_{\rho, \delta}^{m}\left(\Omega \times R_{\xi}\right)\right)
$$

if $D_{t, t^{\prime}}^{j} K\left(x, \xi ; t, t^{\prime}\right) \in \mathscr{E}^{\mathscr{E}^{0}}\left(\Lambda ; S_{\rho, \delta}^{m}\left(\Omega \times R_{\xi}\right)\right)$ for all $j, 0 \leqq j \leqq p$, where $D_{t, t^{\prime}}^{j}$, denotes a differential operator of the form

$$
\frac{\partial^{j}}{\partial t^{j_{1}} \partial t^{j_{2}}}, j_{1}+j_{2}=j
$$


We set

$$
\mathscr{E}\left(\Lambda ; S_{\rho, \delta}^{m}\left(\Omega_{,} \times R_{\xi}\right)\right)=\bigcap_{p \geqq 0} \mathscr{E}^{p}\left(\Lambda ; S_{\rho, \delta}^{m}\left(\Omega \times R_{\xi}\right)\right) .
$$

After some shrinking of the sets $\Omega$ and $I$ (rewrite them $\Omega$ and $I$ ) we have

Proposition 4.1. For any $\varepsilon>0$ we have

$$
\begin{aligned}
& K_{0}\left(x, \xi ; t, t^{\prime}\right) \in \mathscr{E}\left(\Delta ; S^{-\infty}\left(\Omega \times R_{\xi}\right)\right) \cap \bigcap_{p \geqq 0} \mathscr{E}^{p}\left(\bar{\Delta} ; S_{1,2 \ell /(2 \ell+1)}^{\varepsilon+2 p}\left(\Omega \times R_{\xi}\right)\right), \\
& \left|\left(K_{0}\left(x, \xi ; t, t^{\prime}\right)-1\right)(1+|\xi|)^{-\varepsilon}\right| \rightrightarrows 0 \text { in } \Omega \times R_{\xi} \text { as } t \downarrow t^{\prime}, \\
& \left|D_{t, t^{\prime}}^{p} D_{x}^{\beta} D_{\xi}^{\alpha} K_{0}(1+|\xi|)^{(-\beta \times 2 \ell) /(2 \ell+1)-2 p+\alpha-\varepsilon}\right| \rightrightarrows 0 \\
& \text { in } \Omega \times R_{\xi} \text { as } t \downarrow t^{\prime} \text {, if } 2 p<\alpha \text {. } \\
& (\rightarrow \text { means uniform convergency.) }
\end{aligned}
$$

Proof. We may assume

$$
\delta\left(t-t^{\prime}\right)^{2 \ell+1} \leqq \operatorname{Re} \int_{t^{\prime}}^{t} a(x, \tau) d \tau, \quad\left(x, t, t^{\prime}\right) \in \Omega \times \bar{\Delta}
$$

for some $\delta>0$. (See Lemma 2.2.) That is, we have

$$
\delta\left(t-t^{\prime}\right)^{2 \ell+1} \xi^{2} \leqq \operatorname{Re} \int_{t^{\prime}}^{t} a(x, \tau) \xi^{2} d \tau, \quad\left(x, \xi ; t, t^{\prime}\right) \in \Omega \times R_{\xi} \times \bar{\Delta}
$$

Remembering that

$$
K_{0}\left(x, \xi ; t, t^{\prime}\right)=\left\{\begin{array}{l}
\exp \left(-\int_{t^{\prime}}^{t} a(x, \tau) \xi^{2} d \tau\right) \quad\left(x, \xi ; t, t^{\prime}\right) \in \Omega \times R_{\xi} \times \bar{\Delta} \\
0 \quad t<t^{\prime}
\end{array}\right.
$$

we see

$$
K_{0}\left(x, \xi ; t, t^{\prime}\right) \in \mathscr{E}\left(\Delta ; S^{-\infty}\left(\Omega \times R_{\xi}\right)\right) .
$$

Next we shall prove

$$
K_{0} \in \bigcap_{p \geqq 0} \mathscr{E}^{p}\left(\bar{\Delta} ; S_{1,2 \ell(2 \ell+1)}^{\varepsilon+2 p}\left(\Omega \times R_{\xi}\right)\right), \quad \varepsilon>0
$$

in several steps.

(i) We have

$$
\left|K_{0}\left(x, \xi ; t, t^{\prime}\right)\right| \leqq 1 \quad \text { in } \Omega \times R_{\xi} \times \bar{\Delta},
$$

$$
\left|\left(K_{0}\left(x, \xi ; t, t^{\prime}\right)-1\right)(1+|\xi|)^{-\varepsilon}\right| \rightrightarrows 0 \text { in } \Omega \times R_{\xi} \text { as } t \downarrow t^{\prime} .
$$


In fact, for any compact set $\Xi \subset R_{\xi}$ we have

$$
K_{0}\left(x, \xi ; t, t^{\prime}\right) \rightrightarrows 1 \text { in } \Omega \times \Xi \text { as } t \downarrow t^{\prime}
$$

Hence we have (4.7) for any $\varepsilon>0$.

(ii) Let $\alpha$ be a positive integer. We shall study $D_{\xi}^{\alpha} K_{0}\left(x, \xi ; t, t^{\prime}\right)=$ $\left(\partial^{\alpha} / \partial \xi^{\alpha}\right) K_{0}\left(x, \xi ; t, t^{\prime}\right)$ which is expressed as a linear combination of terms

$$
\begin{gathered}
\left(\prod_{j} D_{\xi}^{\alpha^{(j)}} \int_{t^{\prime}}^{t} a(x, \tau) \xi^{2} d \tau\right) \cdot K_{0}\left(x, \xi ; t, t^{\prime}\right), \\
0<\alpha^{(j)} \leqq 2, \alpha^{(1)}+\cdots+\alpha^{(j)}+\cdots=\alpha .
\end{gathered}
$$

Rewrite (4.8) as follows:

$$
\prod_{j}\left(D_{\xi}^{\alpha(j)} \int_{t^{\prime}}^{t} a(x, \tau) \xi^{2} d \tau\right) \cdot K_{0}\left(x, \xi ; t, t^{\prime}\right)^{\alpha^{(j) / \alpha}}
$$

For a factor with $\alpha^{(j)}=1$ we see by (1.6)

$$
\begin{aligned}
& \left|2 \int_{t^{\prime}}^{t} a(x, \tau) \xi d \tau \cdot \exp \left(-\frac{1}{\alpha} \int_{t^{\prime}}^{t} a(x, \tau) \xi^{2} d \tau\right)\right| \\
& \quad \leqq 2 C|\xi|^{-1} \int_{t^{\prime}}^{t} \operatorname{Re} a(x, \tau) \xi^{2} d \tau \cdot \exp \left(-\frac{1}{\alpha} \int_{t^{\prime}}^{t} \operatorname{Re} a(x, \tau) \xi^{2} d \tau\right) \\
& \quad \leqq C^{\prime}|\xi|^{-1}
\end{aligned}
$$

For a factor with $a^{(j)}=2$ we see

$$
\begin{aligned}
& \left|2 \int_{t^{\prime}}^{t} a(x, \tau) d \tau \cdot \exp \left(-\frac{2}{\alpha} \int_{t^{\prime}}^{t} a(x, \tau) \xi^{2} d \tau\right)\right| \\
& \quad \leqq C|\xi|^{-2} \int_{t^{\prime}}^{t} \operatorname{Re} a(x, \tau) \xi^{2} d \tau \cdot \exp \left(-\frac{2}{\alpha} \int_{t^{\prime}}^{t} \operatorname{Re} a(x, \tau) \xi^{2} d \tau\right) \\
& \quad \leqq C^{\prime}|\xi|^{-2} .
\end{aligned}
$$

We use the same symbols $C, C^{\prime}, \cdots$ to express the different constants. Thus we have

$$
\left|D_{\xi}^{\alpha} K_{0}\left(x, \xi ; t, t^{\prime}\right)\right| \leqq C_{\alpha}(1+|\xi|)^{-\alpha} \quad \text { in } \Omega \times R_{\hat{\xi}} \times \bar{\Delta} .
$$

Furthermore, as in the step (i), from (4.6), (4.8) and (4.10) we have

$$
\left|D_{\xi}^{\alpha} K_{0}\left(x, \xi ; t, t^{\prime}\right)(1+|\xi|)^{\alpha-\varepsilon}\right| \rightrightarrows 0 \text { in } \Omega \times R_{\xi} \text { as } t \downarrow t^{\prime}
$$

for any $\varepsilon>0$.

(iii) For a positive integer $\beta, D_{x}^{\beta} K_{0}=\left(\partial^{\beta} / \partial x^{\beta}\right) K_{0}\left(x, \xi ; t, t^{\prime}\right)$ is expressed as a linear combination of terms 


$$
\begin{gathered}
\left(\prod_{j} D_{x}^{\beta^{(j)}} \int_{t^{\prime}}^{t} a(x, \tau) \xi^{2} d \tau\right) \cdot \exp \left(-\int_{t^{\prime}}^{t} a(x, \tau) \xi^{2} d \tau\right) \\
0<\beta^{(j)}, \beta^{(1)}+\cdots+\beta^{(j)}+\cdots=\beta .
\end{gathered}
$$

As in the step (ii) rewrite (4.12) as follows:

$$
\prod_{j}\left(D_{x}^{\beta^{(j)}} \int_{t^{\prime}}^{t} a(x, \tau) \xi^{2} d \tau\right) \cdot \exp \left(-\frac{\beta^{(j)}}{\beta} \int_{t^{\prime}}^{t} a(x, \tau) \xi^{2} d \tau\right) .
$$

First we investigate a factor with $\beta^{(j)}=1$ :

$$
\int_{t^{\prime}}^{t} a_{x}(x, \tau) \xi^{2} d \tau \cdot \exp \left(-\frac{1}{\beta} \int_{t^{\prime}}^{t} a(x, \tau) \xi^{2} d \tau\right) \text {. }
$$

By virtue of (1.7) and Lemma 2.1 we have

$$
\begin{aligned}
\left|\int_{t^{\prime}}^{t} a_{x}(x, \tau) \xi^{2} d \tau\right| & \leqq C \xi^{2} \int_{t^{\prime}}^{t}(\operatorname{Re} a(x, \tau))^{1 / 2} d \tau \\
& \leqq C|\xi|\left(t-t^{\prime}\right)^{1 / 2}\left(\int_{t^{\prime}}^{t} \operatorname{Re} a(x, \tau) \xi^{2} d \tau\right)^{1 / 2} \\
& \leqq C^{\prime} \mid \xi \xi^{2 \ell /(2 \ell+1)}\left(\xi^{2}\left(t-t^{\prime}\right)^{2 \ell+1}\right)^{1 / 2(2 \ell+1)} \cdot\left(\int_{t^{\prime}}^{t} \operatorname{Re} a(x, \tau) \xi^{2} d \tau\right)^{1 / 2}
\end{aligned}
$$

Hence by using Lemma 2.2 we have

$$
|(4.14)| \leqq C^{\prime \prime}(1+|\xi|)^{2 \ell /(2 \ell+1)}
$$

Next consider a factor with $\beta^{(j)} \geqq 2$ :

$$
\begin{aligned}
\left|\int_{t^{\prime}}^{t} D_{x}^{\beta(j)} a(x, \tau) \xi^{2} d \tau\right| & \leqq C \xi^{2}\left(t-t^{\prime}\right) \\
& \leqq C|\xi|^{(2 \times 2 \ell) /(2 \ell+1)}\left(\xi^{2}\left(t-t^{\prime}\right)^{2 \ell+1}\right)^{1 /(2 \ell+1)}
\end{aligned}
$$

Hence we have as above

$$
\begin{aligned}
& \left|\int_{t^{\prime}}^{t} D_{x}^{\beta^{(j)}} a(x, \tau) \xi^{2} d \tau \cdot \exp \left(-\frac{\beta^{(j)}}{\beta} \int_{t^{\prime}}^{t} a(x, \tau) \xi^{2} d \tau\right)\right| \\
& \leqq C^{\prime}(1+|\xi|)^{\left(\beta^{(j)} \times 2 \ell\right) /(2 \ell+1)}, \quad\left(\beta^{(j)} \geqq 2\right) .
\end{aligned}
$$

Thus we have for any $\beta>0$

$$
\left|D_{x}^{\beta} K_{0}\left(x, \xi ; t, t^{\prime}\right)\right| \leqq C(1+|\xi|)^{(\beta \times 2 \ell) /(2 \ell+1)} \quad \text { in } \Omega \times R_{\xi} \times \bar{\Delta} .
$$

Furthermore as in (i), from (4.12) and (4.17) we have

$$
\begin{gathered}
\left|D_{x}^{\beta} K_{0}\left(x, \xi ; t, t^{\prime}\right)(1+|\xi|)^{(-\beta \times 2 \ell) /(2 \ell+1)-\varepsilon}\right| \rightrightarrows 0 \\
\text { in } \Omega \times R_{\xi} \text { as } t \downarrow t^{\prime}
\end{gathered}
$$

for any $\varepsilon>0$. 
(iv) We shall study $D_{x}^{\beta} D_{\xi}^{\alpha} K_{0}\left(x, \xi ; t, t^{\prime}\right)(\alpha+\beta>0)$ which is expressed as a linear combination of terms

$$
\begin{aligned}
& \left(\prod_{i, j} D_{x}^{\beta^{(j)}} D_{\xi}^{\alpha^{(i)}} \int_{t^{\prime}}^{t} a(x, \tau) \xi^{2} d \tau\right) \cdot K_{0}\left(x, \xi ; t, t^{\prime}\right) \\
& \quad=\prod_{i, j}\left(D_{x}^{\beta^{(j)}} D_{\xi}^{\alpha^{(i)}} \int_{t^{\prime}}^{t} a(x, \tau) \xi^{2} d \tau \cdot K_{0}\left(x, \xi ; t, t^{\prime}\right)^{\left(\beta^{(j)}+\alpha^{(i)}\right) /(\beta+\alpha)}\right), \\
& 0 \leqq \alpha^{(i)} \leqq 2, \quad 0<\beta^{(j)}+\alpha^{(i)}, \quad \sum_{i, j}\left(\beta^{(j)}+\alpha^{(i)}\right)=\beta+\alpha .
\end{aligned}
$$

There are three cases in the factors in (4.19): $\alpha^{(i)}=0, \alpha^{(i)}=1$ and $\alpha^{(i)}=2$.

(a) For the factors with $\alpha^{(i)}=0$, we have examined in the step (iii).

(b) For the factors with $\alpha^{(i)}=1$ we have to examine the cases $\beta^{(j)}=1$ and $\beta^{(j)} \geqq 2$.

(b.1) Case $\alpha^{(i)}=1, \beta^{(j)}=1$ :

$$
\begin{aligned}
\left|\xi \int_{t^{\prime}}^{t} a_{x}(x, \tau) d \xi\right| & \leqq|\xi|\left(t-t^{\prime}\right)^{1 / 2}\left(\int_{t^{\prime}}^{t} \operatorname{Re} a(x, \tau) d \tau\right)^{1 / 2} \\
& =\mid \xi^{2 \ell /(2 \ell+1)-1}\left(\xi^{2}\left(t-t^{\prime}\right)^{2 \ell+1}\right)^{1 / 2(2 \ell+1)}\left(\int_{t^{\prime}}^{t} \xi^{2} \operatorname{Re} a(x, \tau) d \tau\right)^{1 / 2} .
\end{aligned}
$$

By using this inequality we have as before

$$
\begin{gathered}
\left|D_{x} D_{\xi} \int_{t^{\prime}}^{t} a(x, \tau) \xi^{2} d \tau \cdot K_{0}^{2 /(\alpha+\beta)}\right| \leqq C(1+|\xi|)^{2 \ell /(2 \ell+1)-1} \\
\text { in } \Omega \times R_{\xi} \times \bar{\Delta} .
\end{gathered}
$$

(b.2) $\quad$ Case $\alpha^{(i)}=1, \beta^{(j)} \geqq 2$ :

$$
\begin{aligned}
\left|\xi \int_{t^{\prime}}^{t} D_{x}^{\beta(j)} a(x, \tau) d \tau\right| & \leqq C|\xi|\left(t-t^{\prime}\right) \\
& =C|\xi|^{(2 \times 2 \ell) /(2 \ell+1)-1}\left(\xi^{2}\left(t-t^{\prime}\right)^{2 \ell+1}\right)^{1 /(2 \ell+1)} .
\end{aligned}
$$

By using this inequality we have

$$
\begin{gathered}
\left|D_{x}^{\beta^{(j)}} D_{\xi} \int_{t^{\prime}}^{t} a(x, \tau) \xi^{2} d \tau \cdot K_{0}^{\left(1+\beta^{(j)}\right) /(\alpha+\beta)}\right| \leqq C(1+|\xi|)^{\left(\beta^{(j)} \times 2 \ell\right) /(2 \ell+1)-1} \\
\text { in } \Omega \times R_{\xi} \times \bar{\Delta}
\end{gathered}
$$

(c) For the factors with $\alpha^{(i)}=2$ we also have to examine the cases $\beta^{(j)}=1$ and $\beta^{(j)} \geqq 2$.

(c.1) Case $\alpha^{(i)}=2, \beta^{(j)}=1$ : 


$$
\begin{aligned}
\left|\int_{t^{\prime}}^{t} a_{x}(x, \tau) d \tau\right| & \leqq C\left(t-t^{\prime}\right)^{1 / 2}\left(\int_{t^{\prime}}^{t} \operatorname{Re} a(x, \tau) d \tau\right)^{1 / 2} \\
& =C|\xi|^{2 \ell /(2 \ell+1)-2}\left(\xi^{2}\left(t-t^{\prime}\right)^{2 \ell+1}\right)^{1 / 2(2 \ell+1)}\left(\int_{t^{\prime}}^{t} \operatorname{Re} a(x, \tau) \xi^{2} d \tau\right)^{1 / 2} .
\end{aligned}
$$

Thus we have

$$
\begin{gathered}
\left|D_{x} D_{\xi}^{2} \int_{t^{\prime}}^{t} a(x, \tau) \xi^{2} d \tau \cdot K_{0}^{3 /(\alpha+\beta)}\right| \leqq C(1+|\xi|)^{2 \ell /(2 \ell+1)-2} \\
\text { in } \Omega \times R_{\xi} \times \bar{\Delta} .
\end{gathered}
$$

(c.2) $\quad$ Case $\alpha^{(i)}=2, \beta^{(j)} \geqq 2$ :

$$
\begin{aligned}
\left|D_{x}^{\beta^{(j)}} \int_{t^{\prime}}^{t} a(x, \tau) d \tau\right| & \leqq C\left(t-t^{\prime}\right) \\
& =C|\xi|^{(2 \times 2 \ell) /(2 \ell+1)-2}\left(\xi^{2}\left(t-t^{\prime}\right)^{2 \ell+1}\right)^{1 /(2 \ell+1)}
\end{aligned}
$$

from where we have

$$
\begin{gathered}
\left|D_{x}^{\beta^{(j)}} D_{\xi}^{2} \int_{t^{\prime}}^{t} a(x, \tau) \xi^{2} d \tau \cdot K_{0}^{\left(2+\beta^{(j)} /(\alpha+\beta)\right.}\right| \leqq C(1+|\xi|)^{\left(\beta^{(j)} \times 2 \ell\right) /(2 \ell+1)-2} \\
\text { in } \Omega \times R_{\xi} \times \bar{\Delta} .
\end{gathered}
$$

Combining all the above investigation we have finally

$$
\left|D_{x}^{\beta} D_{\xi}^{\alpha} K_{0}\left(x, \xi ; t, t^{\prime}\right)\right| \leqq C_{\alpha, \beta}(1+\mid \xi)^{(\beta \times 2 \ell) /(2 \ell+1)-\alpha} \quad \text { in } \Omega \times R_{\xi} \times \bar{\Delta}
$$

for all integers $\alpha, \beta \geqq 0$.

Furthermore from (4.19) and (4.24) we have

$$
\begin{gathered}
\left|D_{x}^{\beta} D_{\xi}^{\alpha} K_{0}\left(x, \xi ; t, t^{\prime}\right)(1+|\xi|)^{(-\beta \times 2 \ell) /(2 \ell+1)+\alpha-\varepsilon}\right| \rightrightarrows 0 \\
\text { in } \Omega \times R_{\xi} \text { as } t \downarrow t^{\prime}
\end{gathered}
$$

for any $\varepsilon>0,(\beta+\alpha>0)$.

(d) From (4.19), (4.24) we can easily see

$$
\begin{gathered}
\left|D_{t, t^{\prime}}^{p} D_{x}^{\beta} D_{\xi}^{\alpha} K_{0}\right| \leqq C_{\alpha, \beta, p}(1+|\xi|)^{(\beta \times 2 \ell) /(2 \ell+1)+2 p-\alpha} \\
\text { in } \Omega \times R_{\xi} \times \bar{\Delta} .
\end{gathered}
$$

Furthermore, since $0 \leqq \alpha^{(i)} \leqq 2$ in (4.19) and by the proof of (4.25), we have for any $\varepsilon>0$

$$
\begin{gathered}
\left|D_{t, t^{\prime}}^{p} D_{x}^{\beta} D_{\xi}^{\alpha} K_{0}(1+|\xi|)^{(-\beta \times 2 \ell) /(2 \ell+1)-2 p+\alpha-\varepsilon}\right| \rightrightarrows 0 \\
\text { in } \Omega \times R_{\xi} \text { as } t \downarrow t^{\prime}
\end{gathered}
$$

if $2 p<\alpha$. 
Thus the proof of Proposition 4.1 is completed.

Proposition 4.2. The oscillatory integral (cf. [2]) :

$$
\mathscr{K}_{0}\left(x, y, t, t^{\prime}\right)=\frac{1}{\sqrt{2 \pi}} \int_{-\infty}^{\infty} e^{i(x-y) \xi} K_{0}\left(x, \xi ; t, t^{\prime}\right) d \xi
$$

defines a function in $C^{\infty}(W), W=\left\{\left(x, y, t, t^{\prime}\right) \in \Omega \times R_{y} \times I \times I ;|x-y|+\right.$ $\left.\left|t-t^{\prime}\right|>0\right\}$.

Proof. By definition $\mathscr{K}_{0}\left(x, y, t, t^{\prime}\right)=0$ for $t^{\prime}>t$. By (4.12) we can see

$$
\begin{aligned}
& \left|D_{t, t^{\prime}}^{p} D_{x}^{\beta} D_{y}^{\alpha}\left(e^{i(x-y) \xi} K_{0}\left(x, \xi ; t, t^{\prime}\right)\right)\right| \\
& \quad \leqq C_{\alpha, \beta, p}(1+|\xi|)^{\alpha+2 \beta+2 p} \exp \left(-\delta \xi^{2}\left(t-t^{\prime}\right)^{2 \ell+1}\right)
\end{aligned}
$$

if $t^{\prime}<t$.

Hence $\mathscr{K}_{0}\left(x, y, t, t^{\prime}\right)$ is infinitely differentiable in $\left\{\left(x, y, t, t^{\prime}\right) \in \Omega \times R_{y} \times I\right.$ $\left.\times I ;\left|t-t^{\prime}\right|>0\right\}$.

On the other hand, if $x \neq y, t \geqq t^{\prime}$, we have (in the oscillatory sense)

$$
\begin{aligned}
(x- & y)^{j} \mathscr{K}_{0}\left(x, y, t, t^{\prime}\right) \\
& =\int_{-\infty}^{\infty}\left(\frac{1}{i} \frac{\partial}{\partial \xi}\right)^{j} e^{i(x-y) \xi} \cdot K_{0}\left(x, \xi ; t, t^{\prime}\right) d \xi \\
& =(-1)^{\alpha} \int_{-\infty}^{\infty} e^{i(x-y) \xi}\left(\frac{1}{i} \frac{\partial}{\partial \xi}\right)^{j} K_{0}\left(x, \xi ; t, t^{\prime}\right) d \xi .
\end{aligned}
$$

By Proposition 4.1 it is obviously verified that

$$
\lim _{t \downarrow t^{\prime}} D_{t, t^{\prime}}^{p} D_{x}^{\beta} D_{y}^{\alpha}(x-y)^{j} \mathscr{K}_{0}\left(x, y, t, t^{\prime}\right)=0
$$

if $2 p+(\beta \times 2 \ell) /(2 \ell+1)+\alpha<j-1$. This completes the proof since $j$ is arbitrary.

Proposition 4.3. Let $\mathscr{K}_{0}\left(x, y, t, t^{\prime}\right)$ be as in Proposition 4.2. Then $\mathscr{K}_{0}\left(x, y, t, t^{\prime}\right)$ is regular in $\left(y, t^{\prime}\right)$ as well as in $(x, t)$.

Proof. (i) For $v=v\left(y, t^{\prime}\right) \in C_{0}^{\infty}\left(R_{y} \times I\right)$ consider the integral

$$
\begin{aligned}
\int_{R_{y} \times I} & \mathscr{K}_{0}\left(x, y, t, t^{\prime}\right) v\left(y, t^{\prime}\right) d y d t^{\prime} \\
\quad= & \int_{T^{\prime}}^{t}\left(\int_{-\infty}^{\infty} e^{i x \xi} K_{0}\left(x, \xi ; t, t^{\prime}\right) \hat{v}\left(\xi, t^{\prime}\right) d \xi\right) d t^{\prime} .
\end{aligned}
$$

By using the fact that 


$$
\left|\hat{v}\left(\xi, t^{\prime}\right)\right| \leqq C_{N}(1+|\xi|)^{-N} \quad N \geqq 0
$$

and by Proposition 4.1 we can see this integral defines a function in $C^{\infty}\left(\Omega_{x} \times I_{t}\right)$.

(ii) To prove the regularity in $\left(y, t^{\prime}\right)$ we need the following lemma.

LEMMA 4.4 (cf. [1], Lemma 2.3.) Let $\Omega$ be an open set in $R_{x}^{n}$. Let $a(x, \xi) \in S_{\rho, \delta}^{m}\left(\Omega \times R_{\xi}^{n}\right)$ and $v(x) \in C_{0}^{\infty}(\Omega)$. Then we have

$$
\left|\int e^{i x \xi} a(x, \xi) v(x) d x\right| \leqq C_{N}(1+|\xi|)^{m+\delta N-N}, \quad \xi \in R_{\xi}^{n},
$$

where $N$ is an arbitrary positive integer.

Now let $\psi(x, t) \in C_{0}^{\infty}(\Omega \times I)$ and consider the integral

$$
\begin{aligned}
\int_{\Omega} \mathscr{K}_{0}\left(x, y, t, t^{\prime}\right) \psi(x, t) d x \\
\quad=\int_{\Omega}\left(\int_{-\infty}^{\infty} e^{i(x-y) \xi} K_{0}\left(x, \xi ; t, t^{\prime}\right) d \xi\right) \psi(x, t) d x \\
\quad=\int_{-\infty}^{\infty} e^{-i y \xi}\left(\int_{\Omega} e^{i x \xi} K_{0}\left(x, \xi ; t, t^{\prime}\right) \psi(x, t) d x\right) d \xi .
\end{aligned}
$$

We set

$$
F_{0}\left(\xi ; t, t^{\prime}\right)=\int e^{i x \xi} K_{0}\left(x, \xi ; t, t^{\prime}\right) \psi(x, t) d x
$$

Then by Lemma 4.4 and Proposition 4.1 we have $F_{0}\left(\xi ; t, t^{\prime}\right) \in \mathscr{E}\left(\bar{\Delta} ; S^{-\infty}\right)$. Whence we have

$$
\begin{aligned}
\int_{\Omega \times I} & \mathscr{K}_{0}\left(x, y, t, t^{\prime}\right) \psi(x, t) d x d t \\
\quad & =\int_{t^{\prime}}^{T_{2}} \int_{-\infty}^{\infty} e^{-i y \xi} F_{0}\left(\xi ; t, t^{\prime}\right) d \xi d t \in C^{\infty}\left(R_{y} \times I_{t^{\prime}}\right) .
\end{aligned}
$$

This completes the proof of Proposition 4.3. (cf. Lemma 5.2.)

\section{§5. Symbols}

$K_{j}\left(x, \xi ; t, t^{\prime}\right), j=0,1,2, \cdots$ We recall some notations :

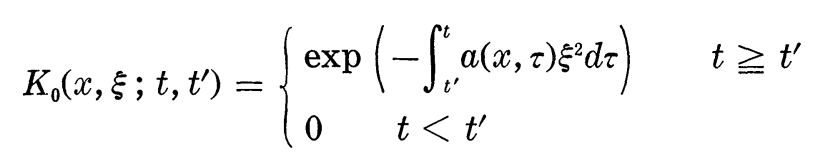




$$
\begin{aligned}
L_{1}= & \frac{\partial}{\partial x}+a(x, t) \xi^{2}, \\
L_{2}= & -2 i a(x, t) \xi \frac{\partial}{\partial x}-a(x, t) \frac{\partial^{2}}{\partial x^{2}}+b(x, t) \frac{\partial}{\partial x} \\
& +i \xi b(x, t)+c(x, t),
\end{aligned}
$$

For $j=0,1,2, \cdots$

$$
K_{j+1}\left(x, \xi ; t, t^{\prime}\right)=\left\{\begin{array}{l}
\int_{t^{\prime}}^{t} \exp \left(-\int_{s}^{t} a(x, \tau) \xi^{2} d \tau\right) \cdot L_{2} K_{j}\left(x, \xi ; s, t^{\prime}\right) d s, \quad t \geqq t^{\prime} \\
0 \quad t \leqq t^{\prime}
\end{array}\right.
$$

Proposition 5.1. For any $\varepsilon>0$ it holds that

$$
\begin{gathered}
K_{j}\left(x, \xi ; t, t^{\prime}\right) \in \bigcap_{p \geqq 0} \mathscr{E}^{p}\left(\bar{\Delta} ; S_{1,2 \ell /(2 \ell+1)}^{\varepsilon+2 p-(j / 2(2 \ell+1))}\left(\Omega \times R_{\xi}\right)\right), \quad j=0,1,2, \cdots, \\
\left|D_{t, t}^{p} D_{x}^{\beta} D_{\xi}^{\alpha} K_{j}(1+|\xi|)^{(-\beta \times 2 \ell) /(2 \ell+1)-2 p+\alpha+(j / 2(2 \ell+1))-\varepsilon}\right| \rightrightarrows 0 \\
\text { in } \Omega \times R_{\xi} \text { as } t \downarrow t^{\prime}, \text { if } 0 \leqq p<j .
\end{gathered}
$$

Proof. As in the proof of Proposition 4.1 we shall prove in several steps. We shall start by showing that

$$
K_{j}\left(x, \xi ; t, t^{\prime}\right) \in \mathscr{E}^{\mathscr{0}}\left(\bar{\Delta} ; S_{1,2 \ell /(2 \ell+1)}^{\epsilon-(j / 2 \ell+1))}\left(\Omega \times R_{\xi}\right)\right), \quad j=0,1,2, \cdots .
$$

Assuming the case $j$, we shall show the case $j+1$. The reasoning is very similar to the proof of Proposition 4.1 so we shall only give a sketch.

(i) We have

$$
\left|K_{j+1}\left(x, \xi ; t, t^{\prime}\right)\right| \leqq C(1+|\xi|)^{(-(j+1)) /(2(2 \ell+1))} \quad \text { in } \Omega \times R_{\xi} \times \bar{\Delta},
$$

and

$$
\begin{gathered}
\left|K_{j+1}\left(x, \xi ; t, t^{\prime}\right)(1+|\xi|)^{(j+1) /(2(2 \ell+1))-\varepsilon}\right| \\
\text { in } \Omega \times R_{\xi} \text { as } t \downarrow t^{\prime}
\end{gathered}
$$

for any $\varepsilon>0$.

For example, we shall examine a term in the integral of $K_{j+1}$ :

$$
\begin{aligned}
I & \equiv\left|\int_{t^{\prime}}^{t} \exp \left(-\int_{s}^{t} a(x, \tau) \xi^{2} d \tau\right) i \xi b(x, s) K_{j}\left(x, \xi ; s, t^{\prime}\right) d s\right| \\
& \leqq C_{1}(1+|\xi|)^{(-j) /(2(2 \ell+1))} \int_{t^{\prime}}^{t} \exp \left(-\int_{s}^{t} \operatorname{Re} a(x, \tau) \xi^{2} d \tau\right) \cdot|b(x, s) \xi| d s \\
& \leqq C_{1}^{\prime}(1+|\xi|)^{(-j) /(2(2 \ell+1))} \int_{t^{\prime}}^{t} \exp \left(-\int_{s}^{t} \operatorname{Re} a(x, \tau) \xi^{2} d \tau\right)\left(\operatorname{Re} a(x, s) \xi^{2}\right)^{1 / 2} d s,
\end{aligned}
$$




$$
\begin{aligned}
& \int_{t^{\prime}}^{t} \exp \left(-\int_{s}^{t} \operatorname{Re} a(x, \tau) \xi^{2} d \tau\right) \cdot\left(\operatorname{Re} a(x, s) \xi^{2}\right)^{1 / 2} d s \\
& \leqq\left(\int_{t^{\prime}}^{t} \exp \left(-\int_{s}^{t} \operatorname{Re} a(x, \tau) \xi^{2} d \tau\right) \operatorname{Re} a(x, s) \xi^{2} d s\right)^{1 / 2} \\
& \cdot\left(\int_{t^{\prime}}^{t} \exp \left(-\int_{s}^{t} \operatorname{Re} a(x, \tau) \xi^{2} d \tau\right) d s\right)^{1 / 2}=I I \times I I I, \\
& I I^{2}=1-\exp \left(-\int_{t^{\prime}}^{t} \operatorname{Re} a(x, \tau) \xi^{2} d \tau\right) \leqq 1 \text {, } \\
& I I I^{2}=\int_{t^{\prime}}^{t}(t-s)^{(-1) / 2}(t-s)^{1 / 2} \exp \left(-\int_{s}^{t} \operatorname{Re} a(x, \tau) \xi^{2} d \tau\right) d s \\
& \leqq \int_{t^{\prime}}^{t}(t-s)^{(-1) / 2} d s \sup _{t^{\prime} \leqq s \leqq t}(t-s)^{1 / 2} \exp \left(-\int_{s}^{t} \operatorname{Re} a(x, \tau) \xi^{2} d \tau\right) \\
& \leqq C_{2}(1+|\xi|)^{(-1) /(2 \ell+1)} \text {. } \\
& \text { (cf. §4) }
\end{aligned}
$$

Hence we have

$$
I \leqq C_{3}(1+|\xi|)^{(-(j+1)) /(2(2 \ell+1))} .
$$

By the similar calculations we have (5.3).

Furthermore as in $\S 4$, by definition of $K_{j+1}$ and by (5.3), we have (5.4).

(ii) We have

$$
\left|D_{\xi}^{\alpha} K_{j+1}\left(x, \xi ; t, t^{\prime}\right)\right| \leqq C(1+|\xi|)^{(-(j+1)) /(2(2 \ell+1))-\alpha} \quad \text { in } \Omega \times R_{\xi} \times \bar{\Delta},
$$

and

$$
\left|D_{\xi}^{\alpha} K_{j+1}(1+|\xi|)^{(j+1) /(2(2 \ell+1))+\alpha-\varepsilon}\right| \rightrightarrows 0 \quad \text { in } \Omega \times R_{\xi} \text { as } t \downarrow t^{\prime}
$$

for any $\varepsilon>0$.

To obtain (5.5) and (5.6), we have to estimate each term of the expression

$$
\begin{aligned}
& D_{\xi}^{\alpha} K_{j+1}\left(x, \xi ; t, t^{\prime}\right)=D_{\xi}^{\alpha} \int_{t^{\prime}}^{t} \exp \left(-\int_{s}^{t} a(x, \tau) \xi^{2} d \tau\right) L_{2} K_{j}\left(x, \xi ; s, t^{\prime}\right) d s \\
& \quad=\sum_{\alpha_{1}+\alpha_{2}=\alpha} C_{\alpha_{1}, \alpha_{2}} \int_{t^{\prime}}^{t} D_{\xi}^{\alpha_{1}} \dot{\operatorname{xxp}}\left(-\int_{s}^{t} a(x, \tau) \xi^{2} d \tau\right) D_{\xi}^{\alpha_{2}} L_{2} K_{j}\left(x, \xi ; s, t^{\prime}\right) d s \\
& \quad=\sum_{\alpha_{1}+\alpha_{2}=\alpha} C_{\alpha_{1}, \alpha_{2}} \cdot I_{\alpha_{1}, \alpha_{2}} .
\end{aligned}
$$

By the similar calculation as in the step (i) we have

$$
\begin{aligned}
\left|I_{\alpha_{1}, \alpha_{2}}\right| & \leqq C(1+|\xi|)^{-\alpha_{1}} \int_{s}^{t} \exp \left(-\gamma \int_{s}^{t} \operatorname{Re} a(x, \tau) \xi^{2} d \tau\right) \cdot\left|D_{\xi}^{\alpha_{2}} L_{2} K_{j}\left(x, \xi ; s, t^{\prime}\right)\right| d s \\
& =C(1+|\xi|)^{-\alpha_{1}} \cdot I_{\alpha_{2}}
\end{aligned}
$$


for some constant $\gamma>0$ and we have

$$
I_{\alpha_{2}} \leqq C^{\prime}(1+|\xi|)^{-\alpha_{2}-(j+1) /(2(2 \ell+1))} .
$$

Hence we have (5.5) and by the above expression of $D_{\xi}^{\alpha} K_{j+1}$ and by (5.5) we obtain (5.6).

(iii) We have

$$
\begin{gathered}
\left|D_{x}^{\beta} K_{j+1}\left(x, \xi ; t, t^{\prime}\right)\right| \leqq C(1+|\xi|)^{(\beta \times 2 \ell) /(2 \ell+1)-(j+1)(2(2 \ell+1))} \\
\text { in } \Omega \times R_{\xi} \times \bar{\Delta},
\end{gathered}
$$

and

$$
\begin{gathered}
\left|D_{x}^{\beta} K_{j+1}(1+|\xi|)^{-(\beta \times 2 \ell) /(2 \ell+1)+(j+1) / 2(2(2 \ell+1))-\varepsilon}\right| \rightrightarrows 0 \\
\text { in } \Omega \times R_{\xi} \text { as } t \downarrow t^{\prime}
\end{gathered}
$$

for any $\varepsilon>0$.

To obtain (5.8) and (5.9), we have to examine each term in the expression

$$
\begin{aligned}
D_{x}^{\beta} K_{j+1} & =D_{x}^{\beta} \int_{t^{\prime}}^{t} \exp \left(-\int_{s}^{t} a(x, \tau) \xi^{2} d \tau\right) L_{2} K_{j}\left(x, \xi ; s, t^{\prime}\right) d s \\
& =\sum_{\beta_{1}+\beta_{2}=\beta} C_{\beta_{1}, \beta_{2}} \int_{t^{\prime}}^{t} D_{x}^{\beta_{1}} \exp \left(-\int_{s}^{t} a(x, \tau) \xi^{2} d \tau\right) \cdot D_{x}^{\beta_{2}} L_{2} K_{j}\left(x, \xi ; s, t^{\prime}\right) d s .
\end{aligned}
$$

Obviously there are two constants $C, \gamma>0$ such that

$$
\begin{aligned}
\left|\int_{t^{\prime}}^{t} D_{x}^{\beta_{1}} \exp \left(-\int_{s}^{t} a(x, \tau) \xi^{2} d \tau\right) D_{x}^{\beta_{2}} L_{2} K_{j}\left(x, \xi ; s, t^{\prime}\right) d s\right| \\
\quad \leqq C(1+|\xi|)^{\left(\beta_{1} \times 2 \ell\right) /(2 \ell+1)} \int_{t^{\prime}}^{t} \exp \left(-\gamma \int_{s}^{t} a(x, \tau) \xi^{2} d \tau\right)||_{x}^{\beta_{2}} L_{2} K_{j}\left(x, \xi ; s, t^{\prime}\right) \mid d s \\
\quad=C(1+|\xi|)^{\left(\beta_{1} \times 2 \ell\right) /(2 \ell+1)} \cdot I .
\end{aligned}
$$

Therefore we have to show

$$
I \leqq C_{1}(1+|\xi|)^{\left(\beta_{2} \times 2 \ell\right) /(2 \ell+1)-(j+1)(2(2 \ell+1))} \quad \text { in } \Omega \times R_{\xi} \times \bar{\Delta} .
$$

We shall study some terms of the integral $I$ :

$$
\begin{aligned}
\mid \int_{t^{\prime}}^{t} \exp & \left(-\gamma \int_{s}^{t} \operatorname{Re} a(x, \tau) \xi^{2} d \tau\right) \cdot D_{x}^{\beta_{2}}\left(\xi a(x, s) D_{x} K_{j}\left(x, \xi ; s, t^{\prime}\right)\right) d s \mid \\
\leqq & C_{2}\left(\left|\int_{t^{\prime}}^{t} \exp \left(-\gamma \int_{s}^{t} \operatorname{Re} a(x, \tau) \xi^{2} d \tau\right) \cdot \xi a(x, s) D_{x}^{\beta_{2}+1} K_{j}\left(x, \xi ; s, t^{\prime}\right) d s\right|\right. \\
& \quad+\left|\int_{t^{\prime}}^{t} \exp \left(-\gamma \int_{s}^{t} \operatorname{Re} a(x, \tau) \xi^{2} d \tau\right) \cdot \xi a_{x}(x, s) D_{x}^{\beta_{2}} K_{j}\left(x, \xi ; s, t^{\prime}\right) d s\right| \\
& \left.\quad+\left|\int_{t^{\prime}}^{t} \exp \left(-\gamma \int_{s}^{t} \operatorname{Re} a(x, \tau) \xi^{2} d \tau\right) \cdot \xi a_{x x}(x, s) D_{x}^{\beta_{2}-1} K_{j}\left(x, \xi ; s, t^{\prime}\right) d s\right|+\cdots\right) \\
= & C_{2}\left(I^{\prime}+I I^{\prime}+I I I^{\prime}\right),
\end{aligned}
$$




$$
\begin{aligned}
I^{\prime} \leqq & C^{\prime}(1+|\xi|)^{\left(\left(\beta_{2}+1\right) \times 2 \ell\right) /(2 \ell+1)-j /(2(2 \ell+1))-1} \int_{t^{\prime}}^{t} \exp \left(-\gamma \int_{s}^{t} \operatorname{Re} a(x, \tau) \xi^{2} d \tau\right) \\
& \cdot \operatorname{Re} a(x, s) \xi^{2} d s \\
\leqq & C^{\prime}(1+|\xi|)^{\left(\beta_{2} \times 2 \ell\right) /(2 \ell+1)-(j+1) /(2(2 \ell+1))}, \\
I I^{\prime} \leqq & C^{\prime \prime}(1+|\xi|)^{\left(\beta_{2} \times 2 \ell\right) /(2 \ell+1)-j /(2(2 \ell+1))}\left|\int_{t^{\prime}}^{t} \exp \left(-\gamma \int_{s}^{t} a(x, \tau) \xi^{2} d \tau\right) \operatorname{Re} a_{x}(x, s) \xi d s\right| \\
\leqq & C_{1}^{\prime \prime}(1+|\xi|)^{\left(\beta_{2} \times 2 \ell\right) /(2 \ell+1)-j /(2(2 \ell+1))} \int_{t^{\prime}}^{t} \exp \left(-\gamma \int_{s}^{t} a(x, \tau) \xi^{2} d \tau\right)\left(\operatorname{Re} a(x, s) \xi^{2}\right)^{1 / 2} d s \\
\leqq & C_{2}^{\prime \prime}(1+|\xi|)^{\left(\beta_{2} \times 2 \ell\right) /(2 \ell+1)-j /(2(2 \ell+1))}(1+|\xi|)^{(-1) /(2(2 \ell+1))}, \\
& (\operatorname{cf} .(\mathrm{i})) \\
I I I^{\prime} \leqq & C^{\prime \prime \prime}(1+|\xi|)^{\left(\left(\beta_{2}-1\right) \times 2 \ell\right) /(2 \ell+1)-j /(2(2 \ell+1))+1} \int_{t^{\prime}}^{t} \exp \left(-\gamma \int_{s}^{t} a(x, \tau) \xi^{2} d \tau\right) d s, \\
\int_{t^{\prime}}^{t} & \exp \left(-\gamma \int_{s}^{t} a(x, \tau) \xi^{2} d \tau\right) d s \leqq C_{1}^{\prime \prime \prime}(1+|\xi|)^{(-3) /(2(2 \ell+1))} .
\end{aligned}
$$

(cf. (i))

For other terms we can treat in the similar manner and we obtain (5.8), then by (5.8) and (5.10) we have (5.9).

(iv) We can treat $D_{x}^{\beta} D_{\xi}^{\alpha} K_{j+1}\left(x, \xi ; t, t^{\prime}\right)$ by the similar way as in the step (iv) of the proof of Proposition 4.1, that is, by the same calculation as above, we can estimate each term in the expression:

$$
\begin{aligned}
D_{x}^{\beta} D_{\xi}^{\alpha} & K_{j+1}\left(x, \xi ; t, t^{\prime}\right) \\
= & \sum_{\substack{0 \leqq k \leqq \beta \\
0 \leqq j \leqq \alpha}} C_{j, k} \int_{t^{\prime}}^{t} D_{x}^{\beta-k} D_{\xi}^{\alpha-j} \exp \left(-\int_{s}^{t} a(x, \tau) \xi^{2} d \tau\right) \\
& \cdot D_{x}^{k} D_{\xi}^{j} L_{2} K_{j}\left(x, \xi ; s, t^{\prime}\right) d s .
\end{aligned}
$$

We obtain the following properties:

$$
\begin{aligned}
\left|D_{x}^{\beta} D_{\xi}^{\alpha} K_{j+1}\left(x, \xi ; t, t^{\prime}\right)\right| & \leqq C(1+|\xi|)^{(\beta \times 2 \ell) /(2 \ell+1)-\alpha-(j+1) /(2(2 \ell+1))} \\
& \text { in } \Omega \times R_{\xi} \times \bar{\Delta},
\end{aligned}
$$

and

$$
\begin{gathered}
\left|D_{x}^{\beta} D_{\xi}^{\alpha} K_{j+1}(1+|\xi|)^{(-\beta \times 2 \ell) /(2 \ell+1)+(j+1) /(2(2 \ell+1))-\varepsilon}\right| \rightrightarrows 0 \\
\text { in } \Omega \times R_{\xi} \text { as } t \downarrow t^{\prime} \text { for any } \varepsilon>0 .
\end{gathered}
$$

Thus we have proved that

$$
K_{j}\left(x, \xi ; t, t^{\prime}\right) \in \mathscr{E}^{\mathscr{0}}\left(\bar{\Delta} ; S_{1,2 \ell /(2 \ell+1)}^{\varepsilon-j /(2(2 \ell+1))}\left(\Omega \times R_{\xi}\right)\right), \quad j=0,1,2, \ldots .
$$


(v) Next we shall study $D_{t, t^{\prime}}^{p} D_{x}^{\beta} D_{\xi}^{\alpha} K_{j}\left(x, \xi ; t, t^{\prime}\right)$. We need the following lemma.

Lemma 5.2. Let $f\left(t, t^{\prime}, s\right)$ be infinitely differentiable function in the set $\left\{\left(t, t^{\prime}, s\right) ; t^{\prime} \leqq s \leqq t\right\}$. Then we have

$$
\begin{aligned}
& D_{t}^{p} \int_{t^{\prime}}^{t} f\left(t, t^{\prime}, s\right) d s=\int_{t^{\prime}}^{t} D_{t}^{p} f\left(t, t^{\prime}, s\right) d s \\
& \quad+\left.\sum_{j=1}^{p-1} \frac{p(p-1) \cdots(p-j+1)}{j !} D_{t}^{p-j} D_{s}^{j-1} f\left(t, t^{\prime}, s\right)\right|_{s=t}, \\
& D_{t^{\prime}}^{q} \int_{t^{\prime}}^{t} f\left(t, t^{\prime}, s\right) d s=\int_{t^{\prime}}^{t} D_{t^{\prime}}^{q} f\left(t, t^{\prime}, s\right) d s \\
& \quad-\left.\sum_{k=1}^{q} \frac{q(q-1) \cdots(q-k+1)}{k !} D_{t^{\prime}}^{q-k} D_{s}^{k-1} f\left(t, t^{\prime}, s\right)\right|_{s=s^{\prime}}, \\
& D_{t^{\prime}}^{q} D_{t}^{p} \int_{t^{\prime}}^{t} f\left(t, t^{\prime}, s\right) d s=\int_{t^{\prime}}^{t} D_{t^{\prime}}^{q} D_{t}^{p} f\left(t, t^{\prime}, s\right) d s \\
& \quad+D_{t^{\prime}}^{q}\left(\left.\sum_{j=1}^{p-1} \frac{p(p-1) \cdots(p-j+1)}{j !} D_{t}^{p-j} D_{s}^{j-1} f\left(t, t^{\prime}, s\right)\right|_{s=t}\right) \\
& \quad-\sum_{k=1}^{q-1} \frac{q(q-1) \cdots(q-k+1)}{h !} D_{t^{\prime}}^{q-k} D_{s}^{q-1}\left(\left.D_{t}^{p} f\left(t, t^{\prime}, s\right)\right|_{s=t^{\prime}}\right) .
\end{aligned}
$$

By an induction in $j$, using Lemma 5.2 and (5.12), we obtain the following estimates:

$$
\begin{aligned}
& \left|D_{t, t^{\prime}}^{p} D_{x}^{\beta} D_{\xi}^{\alpha} K_{j}\left(x, \xi ; t, t^{\prime}\right)\right| \\
& \quad \leqq C_{\alpha, \beta, p}(1+|\xi|)^{(\beta \times 2 \ell) /(2 \ell+1)-\alpha-j /(2(2 \ell+1))+2 p} .
\end{aligned}
$$

The method of calculation is very similar to that used in the step (i) (iv) so we omit the detail.

It remains to prove (5.2). By definition of $K_{j}$ it holds that

$$
K_{j}\left(x, \xi ; t, t^{\prime}\right) \in C^{\infty}\left(\Omega \times R_{\xi} \times \bar{\Delta}\right), \quad j=0,1,2, \cdots
$$

and

$$
\left.D_{t, t^{\prime}}^{p} D_{x}^{\beta} D_{\xi}^{\alpha} K_{j}\left(x, \xi ; t, t^{\prime}\right)\right|_{t=t^{\prime}}=0
$$

if $p<j$. Thus we have (5.2) by virtue of (5.18).

Q.E.D.

\section{§ 6. Parametrices}

As in $\S 4$ we consider the oscillatory integrals: 
(6.1) $\mathscr{K}_{j}\left(x, y, t, t^{\prime}\right)=\frac{1}{\sqrt{2 \pi}} \int_{-\infty}^{\infty} e^{i(x-y) \xi} K_{j}\left(x, \xi ; t, t^{\prime}\right) d \xi, \quad j=0,1,2, \cdots$,

$$
F_{j}\left(x, y, t, t^{\prime}\right)=\frac{1}{\sqrt{2 \pi}} \int_{-\infty}^{\infty} e^{i(x-y) \xi} L_{2} K_{j}\left(x, \xi ; t, t^{\prime}\right) d \xi, \quad j=0,1,2, \cdots
$$

Proposition 6.1. $\mathscr{K}_{j}\left(x, y, t, t^{\prime}\right)$ and $F_{j}\left(x, y, t, t^{\prime}\right)$ define functions in $C^{\infty}(W), W=\left\{\left(x, y, t, t^{\prime}\right) \in \Omega \times R_{y} \times I \times I ;|x-y|+\left|t-t^{\prime}\right|>0\right\}$. Furthermore we have

$$
D_{t, t^{\prime}}^{p} D_{x}^{\beta} D_{y}^{\alpha} \mathscr{K}_{j}\left(x, y, t, t^{\prime}\right) \in C^{0}\left(\Omega_{x} \times R_{y} \times I \times I\right)
$$

if $(\beta \times 2 \ell) /(2 \ell+1)+\alpha+2 p<j /(2(2 \ell+1))-1$ and

$$
D_{t, t^{\prime}}^{p} D_{x}^{\beta} D_{y}^{\alpha} F_{j}\left(x, y, t, t^{\prime}\right) \in C^{0}\left(\Omega_{x} \times \Omega_{y} \times I \times I\right)
$$

if $(\beta \times 2 \ell) /(2 \ell+1)+\alpha+2 p+2<j /(2(2 \ell+1))-1$.

Proof. By induction in $j$, we easily obtain the following estimates:

$$
\begin{aligned}
\left|D_{t, t^{\prime}}^{p} D_{x}^{\beta} K_{j}\left(x, \xi ; t, t^{\prime}\right)\right| \leqq & C_{p, \beta, j}(1+|\xi|)^{2 p+2 \beta+4 j} \cdot \exp \left(-\delta\left(t-t^{\prime}\right)^{2 \ell+1} \xi^{2}\right) \\
& \text { in } \Omega \times R_{\xi} \times \bar{\Delta} .
\end{aligned}
$$

By using (6.5), we have the first assertion as in the proof of Proposition 4.2. By (5.2) and (5.18) we have (6.3) and (6.4).

The following proposition is obtained just like as Proposition 4.3.

Proposition 6.2. $\mathscr{K}_{j}\left(x, y, t, t^{\prime}\right)$ is regular in $\left(y, t^{\prime}\right)$ as well as in $(x, t)$.

Now we consider the parametrices. By definition of $K_{j}\left(x, \xi ; t, t^{\prime}\right)$ and $\mathscr{K}_{j}\left(x, y, t, t^{\prime}\right)$ we have

$$
P_{x, t}\left(\sum_{j=0}^{\mu} \mathscr{K}_{j}\left(x, y, t, t^{\prime}\right)\right)=\delta\left(x-y, t-t^{\prime}\right)+F_{\mu}\left(x, y, t, t^{\prime}\right), \quad \mu=0,1,2, \cdots
$$

By Proposition 6.1 and 6.2, we have

(i) $\sum_{j=0}^{\mu} \mathscr{K}_{j}\left(x, y, t, t^{\prime}\right) \in C^{\infty}(W), \mu=0,1,2, \cdots$,

(ii) $\sum_{j=0}^{\mu} \mathscr{K}_{j}\left(x, y, t, t^{\prime}\right)$ is very regular, as a distribution, in the sense of Schwartz [8], $\mu=0,1,2, \cdots$,

(iii) $F_{\mu}\left(x, y, t, t^{\prime}\right)$ becomes smoother in $\Omega \times R_{y} \times I \times I$ according as $\mu$ becomes larger.

Thus we obtain that the operator ${ }^{t} \boldsymbol{P}$ defined by

$$
\iint{ }^{t} P \varphi \cdot \psi d x d t=\iint \varphi \cdot P \psi d x d t, \varphi, \psi \in C_{0}\left(\Omega_{x} \times I_{t}\right)
$$


is hypoelliptic in $\Omega \times I$ (cf. [9]). We can also prove the hypoellipticity of the operator $P$ since a translation of the variable $t$ for the operator ${ }^{t} P$ satisfies the conditions given in $\S 1$.

By the above investigation we have obtained Theorem 1.1.

Remark 1. The case of many variables: It is easily verified that our method can be applied for the following operator:

$$
\frac{\partial}{\partial t}-a(x, t) \sum_{i, j=1}^{n} a_{i j}(x, t) \frac{\partial^{2}}{\partial x_{i} \partial x_{j}}+\sum_{j=1}^{n} b_{j}(x, t) \frac{\partial}{\partial x_{j}}+c(x, t)
$$

where $a(x, t), a_{i j}(x, t), b_{j}(x, t), c(x, t)$ are infinitely differentiable functions in a open set $U=\Omega_{x} \times I_{t}$ of $R_{x}^{n} \times R_{t}^{1}$ and we have

$$
\operatorname{Re} \sum_{i, j=1}^{n} a_{i j}(x, t) \xi_{i} \xi_{j} \geqq \delta|\xi|^{2} \quad(x, t) \in U, \xi \in R^{n}
$$

for a positive constant $\delta$. The functions $a(x, t)$ and $b_{j}(x, t)$ satisfy the analogous conditions $(1.4) \sim(1.8)$ in $U$.

Remark 2. Another example for the case of many variables: Our method can be applied for the following operator.

$$
\frac{\partial}{\partial t}-\sum_{i, j=1}^{n} a_{i j}(x, t) \frac{\partial^{2}}{\partial x_{i} \partial x_{j}}+\sum_{j=1}^{n} b_{j}(x, t) \frac{\partial}{\partial x_{j}}+c(x, t),
$$

where $a_{i j}(x, t), b_{j}(x, t), c(x, t)$ are infinitely differentiable functions in a open set $U=\Omega_{n} \times I_{t}$ of $R_{x}^{n} \times R_{t}^{1}$ and we suppose

$$
\operatorname{Re} \sum_{i, j=1}^{n} a_{i j}(x, t) \xi_{i} \xi_{j} \geqq 0, \quad(x, t) \in U, \xi \in R_{u},
$$

(6.9) for all $x \in \Omega$ and all $\xi \in R^{n}, \xi \neq 0$, the function $t \mapsto \operatorname{Re} \sum_{i, j=1}^{n} a_{i j}(x, t) \xi_{i} \xi_{j}$ has only zeros of even order less than or equal to $2 \ell$ in the interval $I$,

$$
\begin{aligned}
& \left|\operatorname{Im} a_{i j}(x, t) \xi_{i} \xi_{j}\right| \leqq C \operatorname{Re} \sum_{i, j=1}^{n} a_{i j}(x, t) \xi_{i} \xi_{j}, \\
& (x, t) \in U, \quad \xi \in R^{n}, \\
& \left|D_{x}^{\beta} \sum_{i, j=1}^{n} a_{i j}(x, t) \xi_{i} \xi_{j}\right| \leqq C_{\beta} \operatorname{Re} \sum_{i, j=1}^{n} a_{i j}(x, t) \xi_{i} \xi_{j} \\
& \text { for all } \beta,(x, t) \in U \text { and } \xi \in R^{n} \text {, }
\end{aligned}
$$




$$
\begin{aligned}
& \sum_{i=1}^{n}\left|\sum_{j=1}^{n} \frac{\partial}{\partial x_{j}} a_{i j}(x, t) \xi_{i}\right| \leqq C\left(\operatorname{Re} \sum_{i, j=1}^{n} a_{i j}(x, t) \xi_{i} \xi_{j}\right)^{1 / 2} \\
&(x, t) \in U, \quad \xi \in R^{n}, \\
& \sum_{j=1}^{n}\left|b_{j}(x, t) \xi_{j}\right| \leqq C\left(\operatorname{Re} \sum_{i, j=1}^{n} a_{i j}(x, t) \xi_{i} \xi_{j}\right)^{1 / 2} \\
&(x, t) \in U, \quad \xi \in R^{n} .
\end{aligned}
$$

Then the operator (6.7) is hypoelliptic in $U$. In fact we can construct the symbols $K_{j}\left(x, \xi ; t, t^{\prime}\right), j=0,1,2, \cdots$, just as in $\S 3$ and we have

$$
\begin{gathered}
K_{j} \in \mathscr{E}\left(\Delta ; S^{-\infty}\left(\Omega \times R_{\xi}\right)\right) \cap \bigcap_{p \geqq 0} \mathscr{E}^{p}\left(\Delta ; S_{1 /(2 \ell+1), 0}^{\varepsilon+2 p-j /(2 \ell+1))}\left(\Omega \times R_{\xi}^{n}\right)\right) \\
j=0,1,2, \cdots,
\end{gathered}
$$

and so on.

EXAMPLE: The operator

$$
\frac{\partial}{\partial t}-\sum_{j=1}^{n} t^{2 \ell_{j}} \frac{\partial^{2}}{\partial x_{j}^{2}}+\sum_{j=1}^{n} t^{\ell_{j}} \frac{\partial}{\partial x_{j}}+1, \quad \ell_{j} \text { integers, } \geqq 0,
$$

satisfies the above condition in a neighbourhood of the origin of $R_{x}^{n} \times R_{t}^{1}$.

Remark 3. The case of infinite order degeneracy: As an example, we consider the operator:

$$
\frac{\partial}{\partial t}-a(t) \frac{\partial^{2}}{\partial x^{2}}
$$

where $a(t)$ is infinitely differentiable function in the interval $I=(-1$ $<t<1$ ) and we suppose $a(t)>0$ for $t \neq 0$ and $a(0)=0$. Take $\Omega=$ $(-\infty<x<\infty)$ and set

$$
K_{0}\left(x, \xi ; t, t^{\prime}\right)= \begin{cases}\exp \left(-\int_{t^{\prime}}^{t} a(\tau) \xi^{2} d \tau\right) & -1<t^{\prime} \leqq t<1 \\ 0 & -1<t<t^{\prime}<1\end{cases}
$$

Then for any $\varepsilon>0$, we have easily

(a) $K_{0}\left(x, \xi ; t, t^{\prime}\right) \in \varepsilon\left(\Delta ; S^{-\infty}\left(\Omega \times R_{\xi}\right)\right) \cap \bigcap_{p=0} \mathscr{E} p\left(\bar{\Delta} ; S_{1,0}^{\epsilon+2 p}\left(\Omega \times R_{\xi}\right)\right)$,

(b) $\left|D_{t, t^{\prime}}^{p} D_{\xi}^{\alpha} K_{0}(1+|\xi|)^{-2 p+\alpha-\varepsilon}\right| \rightrightarrows 0$ in $\Omega \times R_{\xi}$ as $t \downarrow t^{\prime}$ if $2 p<a$.

Thus we have the hypoellipticity of the operator (6.15) in $\Omega \times I$ and we have the fundamental solution defined by an oscillatory integral: 


$$
\begin{aligned}
\mathscr{K}_{0}\left(x, y, t, t^{\prime}\right) & =(2 \pi)^{(-1) / 2} \int_{-\infty}^{\infty} e^{i(x-y) \xi} K_{0}\left(x, \xi ; t, t^{\prime}\right) d \xi \\
& =\frac{1}{2 \sqrt{\pi} A\left(t, t^{\prime}\right)^{1 / 2}} \exp \left[-\frac{(x-y)^{2}}{4 A\left(t, t^{\prime}\right)}\right], \\
A\left(t, t^{\prime}\right) & =\int_{t^{\prime}}^{t} a(\tau) d \tau, \quad t \geqq t^{\prime}
\end{aligned}
$$

\section{REFERENCES}

[1] Hörmander, L.: Pseudo-differential operators and hypoelliptic equations, Amer. Math. Soc. Symp. Pure Math., 10 (1966), Singular integral operators, 138-183.

[ 2 ] Hörmander, L.: Fourier integral operators, I, Acta Math., 127 (1971), 79-183.

[ 3 ] Hörmander, L.: Hypoelliptic second order differential equations, Acta Math. 119 (1968), 147-171.

[4] Kato, Y.: The hypoellipticity of degenerate parabolic differential operators, Jour. Funct. Analysis, Vol. 7, No. 1 (1971), 116-131.

[5] Mizohata, S.: Hypoellipticité des équations paraboliques, Bull. Soc. Math. France, 85 (1957), 15-50.

[6] Nirenberg, L. and Treves, F.: On local solvability of linear partial differential equations, Part I: Necessary conditions. Comm. Pure Applied Math., Vol.23 (1970), 1-38.

[ 7 ] Treves, F.: A new method of proof of the subelliptic estimates, Comm. Pure Applied Math., Vol. 24 (1971), 71-115.

[ 8 ] Treves, F.: Analytic-hypoelliptic partial differential equations of principal type, Comm. Pure Applied Math., Vol. 24 (1971), 537-570.

[ 9 ] Schwartz, L.: Théorie des distributions, Vol. I, Hermann, Paris, (1957).

Nagoya University 